\title{
Delivering noninvasive prenatal testing in a clinical setting using semiconductor sequencing platform
}

\author{
GAO Yuan ${ }^{1,2^{*}},{\text { XIE } \text { Bin }^{3} \& \text { LIU Rui }}^{4}$ \\ ${ }^{1}$ Department of Biomedical Engineering, Johns Hopkins University, Baltimore, MD 21205, USA; \\ ${ }^{2}$ Med Data Quest Inc., San Diego, CA 92130, USA; \\ ${ }^{3}$ Division of Clinical Genetics, Lieber Institute for Brain Development, Johns Hopkins Medical Campus, Baltimore, MD 21205, USA; \\ ${ }^{4}$ Department of Bioengineering, University of California at San Diego, La Jolla, CA 92093, USA
}

Received May 30, 2014; accepted June 16, 2014; published online June 26, 2014

Citation: Gao Y, Xie B, Liu R. Delivering noninvasive prenatal testing in a clinical setting using semiconductor sequencing platform. Sci China Life Sci, 2014 57: 737-738, doi: 10.1007/s11427-014-4696-0

Fetal chromosomal aneuploidies can lead to fetal loss and major birth defects. The best known chromosomal aneuploidy is Down syndrome, or medically termed trisomy 21 , where the affected individual has an extra full or partial copy of chromosome 21 . The extra copy of genetic material is the culprit for causing developmental problem for affected individuals. Three types of Down syndrome exist: trisomy 21 accounts for $95 \%$ of cases, translocation accounts for about $4 \%$ and mosaicism accounts for about $1 \%$. Less common chromosomal aneuploidies include Edward syndrome (trisomy 18), Patau syndrome (trisomy 13), Turner syndrome (45, X), Klinefelter syndrome (47, XXY), and 47, XYY. Many trisomic fetuses die in utero, and could account for up to $13 \%$ of all stillbirths and neonatal death. Even if the fetus would survive, the extra copy of chromosome 21 will affect the baby's physical and intellectual development into adulthood, causing significant personal, social and economic burden.

The incidence of combined chromosomal abnormalities is as high as one in 160 in the United States or one in 60 in China. Such incidence increases as the mother ages and it could reach as high as $2.5 \%$ with maternal age over 35 in China. The estimated incidence of Down syndrome is between 650 and 1000 live births. Such incidence increases significantly as maternal age increases, affecting one in 350

*Corresponding author (email: ygao24@jhmi.edu) for mothers aged 35, one in 106 at age of 40 and as high as one in 30 at 45 years of age. It is therefore important for fetus with chromosomal abnormalities to be detected, as early as possible. Prenatal testing for chromosomal abnormalities is in common practice. Both screening and diagnostic methods have been deployed. Screening methods involve serum marker analysis and/or ultrasound examinations for parameters such as nuchal translucency. Such methods usually suffer from low sensitivity and specificity. Diagnostic tests usually follow if screening show positive results.

Diagnostic tests traditionally are invasive in nature. Amniocentesis and chorionic villus sampling are two conventional invasive methods for prenatal diagnosis of fetal chromosomal aneuploidies. These two methods are highly accurate; however, they both require invasive sampling of fetal materials and carry the risk of fetal loss. The landmark discovery of cell-free fetal nucleic acids in maternal plasma by Dennis Lo in 1997 opened the door to develop noninvasive prenatal diagnosis methods to detect fetal chromosomal aneuploidies [1]. The robust detection of fetal chromosomal aneuploidies using maternal plasma DNA is challenging, due to the relatively low fractional concentration of circulating fetal DNA. In 2008, the reliable detection of fetal trisomy 21 using massively parallel sequencing of maternal plasma DNA reported by Lo's and Gao's labs [2] set the foundation of noninvasive prenatal testing. Subsequently, 
large scale validation of these observations has been conducted by several groups around the world.

Previously, most studies utilize the sequencing-bysynthesis platform for sequencing maternal plasma DNA for aneuploidy detection. Yuan et al. [3], in a proof-of-concept study, demonstrated that semiconductor sequencing could also be used for noninvasive prenatal detection of fetal chromosomal aneuploidy, with the advantage of a shorter turnaround time. At the time of the study, the authors demonstrated that all 13 chromosomal aneuploidies were correctly identified with a $24-\mathrm{h}$ processing time. Because of the number of samples they processed is very small, only 13 cases, six with trisomy 21, two with trisomy 18 and one with trisomy 13 , and it is not a prospective study, the authors cautioned, however, "a further large-scale study is needed to determine the imprecision of noninvasive fetal aneuploidy detection with this system”. Such a large-scale study was achieved recently.

In a paper published in the Proceedings of the National Academy of Sciences of the United States of America last month, led by the groups of Drs. Liao Can, Liu HaiLiang, Zhang XiaoZhuang and Zhang Kang [4], based on both retrospective (515 subjects) and prospective (1760 subjects) study of a total of 2275 pregnant subjects, it clearly demonstrated that a benchtop semiconductor sequencing platform (SSP) is suitable and effective for non-invasive diagnostic of fetal chromosomal abnormalities with a fast turnaround time at a clinical setting. The strength of this paper over the previous paper is two-fold: first, a very large sample size; second, it contains both retrospective and prospective study design. In the retrospective study, 515 subjects who had full karyotyping results were used in the analysis. All 55 fetal trisomy 21 cases were identified using the SSP with a sensitivity and specificity of $99.94 \%$ and $99.46 \%$. The SSP also detected 16 trisomy 18 cases with $100 \%$ sensitivity and $99.24 \%$ specificity and three trisomy 13 cases with $100 \%$ sensitivity and $100 \%$ specificity. Furthermore, 15 fetuses with sex chromosome aneuploidies (10 45, X, 2 47, XYY, 2 $47, \mathrm{XXX}$, and $147, \mathrm{XXY}$ ) were detected. In the prospective study, 1760 subjects without karyotyping results were analyzed. The SSP analysis detected nine fetuses with trisomy 21 , three with trisomy 18 , three with trisomy 13 , and one with $45, \mathrm{X}$. To confirm the detection, the authors randomly selected six positive T21 samples, and the $45, \mathrm{X}$ sample to perform full karyotyping and confirmed all of them to be true positive.

In conclusion, this is the first large-scale clinical study to systematically identify chromosomal aneuploidies based on cell-free fetal DNA using SSP. It also provides a reliable and cost effective method for large-scale noninvasive screening for chromosomal aneuploidies in a clinical setting. There are two understandable shortcomings of this paper. First, for the prospective arm of the study, there are insufficient cases with validated outcome. Second, because the total number of detected positive samples is small (nine fetuses with trisomy 21 , three with trisomy 18 , three with trisomy 13 , and one with $45, \mathrm{X}$ ), instead of randomly selecting samples, all cases should have a complete karyotyping and report the results.

It is expected that the cost of DNA sequencing will be further reduced in the near future owing to the advancement of sequencing technology, computing power as well as development of new bioinformatics tools. The use of targeted sequencing by a number of commercial vendors would potentially provide a cheaper service, breaking the long sought 200 USD or $1000 \mathrm{RMB}$ in China barrier for routine use. Noninvasive prenatal measurement of fetal genome using cell free DNA in pregnant women blood suggested that the prenatal testing based on cell free-DNA sequencing can be used to detect not only fetal chromosomal aneuploidies, but also many inherited and de novo genetic diseases [5]. Recent regulation on prenatal testing set forth by the Chinese government should be welcomed by the general public. It is understood that standardization of clinical trial, testing procedures, pre-testing counseling, as well as post-testing follow-up would ultimately benefit the well-being of the fetus and pregnant mother.

The authors declare that they have no conflict of interest.

1 Lo YM, Corbetta N, Chamberlain PF, Rai V, Sargent IL, Redman CW, Wainscoat JS. Presence of fetal DNA in maternal plasma and serum. Lancet, 1997, 350: 485487

2 Chiu RW, Chan KC, Gao Y, Lau VY, Zheng W, Leung TY, Foo CH, Xie B, Tsui NB, Lun FM, Zee BC, Lau TK, Cantor CR, Lo YM. Noninvasive prenatal diagnosis of fetal chromosomal aneuploidy by massively parallel genomic sequencing of DNA in maternal plasma. Proc Natl Acad Sci USA, 2008, 105: 2045820463

3 Yuan Y, Jiang F, Hua S, Du B, Hao Y, Ye L, Liu J, Feng K, Huang X, Yi X, Wang W, Yang L, Mu F, Liu C, Liang Y. Feasibility study of semiconductor sequencing for noninvasive prenatal detection of fetal aneuploidy. Clin Chem, 2013, 59: 846849

4 Liao C, Yin AH, Peng CF, Fu F, Yang JX, Li R, Chen YY, Luo DH, Zhang YL, Ou YM, Li J, Wu J, Mai MQ, Hou R, Wu F, Luo H, Li DZ, Liu HL, Zhang XZ, Zhang K. Noninvasive prenatal diagnosis of common aneuploidies by semiconductor sequencing. Proc Natl Acad Sci USA, 2014, 111: 74157420

5 Kitzman JO, Snyder MW, Ventura M, Lewis AP, Qiu R, Simmons LE, Gammill HS, Rubens CE, Santillan DA, Murray JC, Tabor HK, Bamshad MJ, Eichler EE, Shendure J. Noninvasive whole-genome sequencing of a human fetus. Sci Transl Med, 2012, 4: 137

Open Access This article is distributed under the terms of the Creative Commons Attribution License which permits any use, distribution, and reproduction in any medium, provided the original author(s) and source are credited. 